\title{
Chronic-Pain-Associated Astrocytic Reaction in the Spinal Cord Dorsal Horn of Human Immunodeficiency Virus-Infected Patients
}

\author{
Yuqiang Shi, ${ }^{1}$ Benjamin B. Gelman, ${ }^{1,2}$ Joshua G. Lisinicchia, ${ }^{2}$ and Shao-Jun Tang ${ }^{1}$ \\ Departments of ${ }^{1}$ Neuroscience and Cell Biology and ${ }^{2}$ Pathology, University of Texas Medical Branch, Galveston, Texas 77555
}

Studies with animal models have suggested that reaction of glia, including microglia and astrocytes, critically contributes to the development and maintenance of chronic pain. However, the involvement of glial reaction in human chronic pain is unclear. We performed analyses to compare the glial reaction profiles in the spinal dorsal horn (SDH) from three cohorts of sex- and age-matched human postmortem tissues: (1) HIV-negative patients, (2) HIV-positive patients without chronic pain, and (3) HIV patients with chronic pain. Our results indicate that the expression levels of $\mathrm{CD} 11 \mathrm{~b}$ and Iba1, commonly used for labeling microglial cells, did not differ in the three patient groups. However, GFAP and $S 100 \beta$, often used for labeling astrocytes, were specifically upregulated in the SDH of the "painpositive" HIV patients but not in the "pain-negative" HIV patients. In addition, proinflammatory cytokines, TNF $\alpha$ and IL- $1 \beta$, were specifically increased in the SDH of pain-positive HIV patients. Furthermore, proteins in the MAPK signaling pathway, including pERK, pCREB and c-Fos, were also upregulated in the SDH of pain-positive HIV patients. Our findings suggest that reaction of astrocytes in the SDH may play a role during the maintenance phase of HIV-associated chronic pain.

\section{Introduction}

Studies on animal models reveal that reaction of microglia and astrocytes in the spinal dorsal horn (SDH) plays an important role in the development of chronic pain (Garrison et al., 1991; Meller et al., 1994; Watkins et al., 1995b, 1997; Clark et al., 2007b; McMahon and Malcangio, 2009; Milligan and Watkins, 2009; Ren and Dubner, 2010). Activated glia may contribute to the pathogenesis of chronic pain by sensitizing pain-processing (transmission) neurons. One elucidated mechanism by which activated glia sensitize neurons involves the sustained upregulation of proinflammatory factors (Kawasaki et al., 2008; McMahon and Malcangio, 2009; Milligan and Watkins, 2009; Ren and Dubner, 2010). Glia are emerging as promising drug targets to control pathological pain (Ledeboer et al., 2006, 2007; Rolan et al., 2008, 2009; Romero-Sandoval et al., 2008; Milligan and Watkins, 2009; Gao and Ji, 2010). Despite the mounting evidence from animal models, glial reaction in human chronic pain patients has not been unequivocally demonstrated - currently available data are from only one patient (Del Valle et al., 2009).

Over $60 \%$ of HIV patients experience chronic pain such as headaches and joint pain (Hewitt et al., 1997; Mirsattari et al.,

\footnotetext{
Received Nov. 8, 2011; revised May 25, 2012; accepted June 17, 2012.

Author contributions: S.-J.T. designed research; Y.S. and J.G.L. performed research; B.B.G. contributed unpublished reagents/analytic tools; Y.S., B.B.G., and S.-J.T. analyzed data; B.B.G. and S.-J.T. wrote the paper.

This work was supported by University of Texas Medical Branch start-up funds and The Whitehall Foundation

(S.-J.T.), and National Institutes of Health Grants U01-MH-083507 and R24-NS-045491 (B.B.G.).

The authors declare no competing financial interests.

Correspondence should be addressed to Dr. Shao-Jun Tang, Department of Neuroscience and Cell Biology, University of Texas Medical Branch, Galveston, TX 77555-1069. E-mail: shtang@utmb.edu.

DOI:10.1523/JNEUROSCI.5628-11.2012

Copyright $\odot 2012$ the authors $\quad 0270-6474 / 12 / 3210833-08 \$ 15.00 / 0$
}

1999; Evers et al., 2000). The HIV-associated chronic pain is acquired during the progress of other HIV-related symptoms (Singer et al., 1993). In rodent models of HIV-associated chronic pain, glial reaction was observed in the SDH. For example, microglia and astrocytes were activated in the ipsilateral SDH of the neuropathy model created by perineural administration of HIV gp120 (Wallace et al., 2007). Hyperactivated microglia and astrocytes were also observed in the SDH in the pain model generated by intrathecal injection of gp120 (Milligan et al., 2001). Importantly, the glial inhibitor minocycline was able to attenuate intrathecal gp120-induced pain (Ledeboer et al., 2005). Milligan et al. (2001) reported that glial reaction mediated intrathecal gp120induced pain via the expression of proinflammatory cytokines. These and other animal studies strongly suggest that glial reaction in the SDH is crucial for the development of HIV-associated chronic pain. Yet direct evidence from human patients is still missing.

In this study, we compared the profiles of glial reaction in the $\mathrm{SDH}$ from HIV-infected patients who experienced chronic pain (i.e., "pain-positive") and from the HIV patients who did not suffer chronic pain (i.e., "pain-negative"). Our results indicated that astrocytes but not microglia were probably reacted in the $\mathrm{SDH}$ of pain-positive HIV patients. Interestingly, the astrocyte reaction did not occur in the pain-negative HIV patients. These findings identify astrocytes as potential drug targets to treat HIVassociated chronic pain.

\section{Materials and Methods}

Human postmortem tissues. Fifteen male subjects were selected from the autopsy archive of the Texas NeuroAIDS Research Center, which is one unit of the National NeuroAIDS Tissue Consortium (NNTC) (Table 1) (Morgello et al., 2001). Five of 15 subjects were HIV-seronegative con- 
Table 1. Human patient information

\begin{tabular}{|c|c|c|c|c|c|c|c|c|c|c|}
\hline$\overline{\text { Subject }}$ & NNTC ID ${ }^{a}$ & Age & Gender & Race & Latino & HIV infected & Pain symptoms in distal leg ${ }^{b}$ & NNTC neurological diagnosis $^{c}$ & Sural nerve diagnosis & Cause of death \\
\hline 1 & 7101958083 & 46 & Male & White & Yes & No & Not done & $\mathrm{N} / \mathrm{A}^{d}$ & Normal & Pneumonia \\
\hline 2 & 7102268283 & 42 & Male & White & No & No & Not done & N/A & N/A & Lung cancer \\
\hline 3 & 7101198377 & 34 & Male & White & No & No & Not done & $\mathrm{N} / \mathrm{A}$ & Normal & Pulmonary embolus \\
\hline 4 & 7101738266 & 38 & Male & White & No & No & Not done & $\mathrm{N} / \mathrm{A}$ & Normal & Endocarditis \\
\hline 5 & 7101727068 & 58 & Male & White & No & No & Not done & $\mathrm{N} / \mathrm{A}$ & Normal & Pneumonia \\
\hline 6 & 7200188777 & 38 & Male & White & No & Yes & 3 & Unable to assign & Normal & HIV/AIDS \\
\hline 7 & 7200586865 & 36 & Male & White & Yes & Yes & None & No HDSPN & Normal & HIV/AIDS \\
\hline 8 & 7100567477 & 55 & Male & White & No & Yes & None & Unable to assign & Normal & HIV/AIDS \\
\hline 9 & 7200267172 & 44 & Male & White & No & Yes & None & № HDSPN & Normal & HIV/AIDS \\
\hline 10 & 7100696768 & 42 & Male & White & No & Yes & None & № HDSPN & N/A & HIV/AIDS \\
\hline 11 & 7100616568 & 32 & Male & White & No & Yes & 1 & HDSPN possible & Mild neuropathy & HIV/AIDS \\
\hline 12 & 7100147470 & 50 & Male & White & No & Yes & $1,2,3$ & HDSPN definite & Moderate neuropathy & HIV/AIDS \\
\hline 13 & 7100766971 & 49 & Male & Black & No & Yes & $1,2,3$ & HDSPN possible & Moderate neuropathy & HIV/AIDS \\
\hline 14 & 7100626868 & 43 & Male & White & No & Yes & $1,2,3$ & HDSPN definite & Moderate neuropathy & HIV/AIDS \\
\hline 15 & 7100118382 & 37 & Male & White & No & Yes & $1,2,3$ & HDSPN possible & Severe neuropathy & HIV/AIDS \\
\hline
\end{tabular}

${ }^{a}$ NNTC ID, National NeuroAIDS Tissue Consortium identifier for public access.

${ }^{b}$ Key to pain symptoms: 1 , pain/burning; 2, tingling; 3 , numbness.

CHDSPN, HIV-associated distal sensory peripheral neuropathy diagnosed within 6 months prior to death.

${ }^{d} \mathrm{~N} / \mathrm{A}$, Not applicable or not available.

trols. The control subjects had no known history of peripheral neuropathy, myelopathy, or chronic pain; they had a normal sural nerve biopsy obtained postmortem; they died of causes not related to HIV/AIDS. Ten of 15 subjects died with HIV/AIDS. Those subjects were examined prospectively and longitudinally at 6 month intervals according to the NNTC protocol and underwent at least one structured neurological examination within 6 months before death (Morgello et al., 2004). A complete autopsy was performed including removal of the spinal cord and a sample of the distal sural nerve. The samples were frozen at $-80^{\circ} \mathrm{C}$ until being prepared for Western blotting. Five of the subjects with HIV/AIDS had no definitive clinical evidence of HIV-associated distal sensory peripheral neuropathy (HDSPN), had no myelopathy clinically or neuropathologically, had no pain syndrome clinically, and had a neuropathologically normal distal sural nerve. Five HIV-positive subjects had HDSPN clinically, had axonal neuropathy documented neuropathologically in the sural nerve biopsy, and had a clinically documented pain syndrome. The structured neurological examination was performed as described previously (Morgello et al., 2001, 2004). The diagnosis of peripheral neuropathy in postmortem sural nerve biopsies was determined by the neuropathologist using plastic-embedded transversely sliced $1.0-\mu \mathrm{m}$-thick sections stained with toluidine blue. Myelinated nerve fiber density was rated as normal, mildly decreased $(<20 \%$ decrease), moderately decreased $(20-60 \%$ decrease), or severely decreased ( $>60 \%$ decrease). Abnormal specimens were confirmed using transmission electron microscopy to evaluate nonmyelinated nerve fiber populations. Myelopathy was evaluated by neuropathologists in transverse sections of lumbar spinal cords fixed in formalin, embedded in paraffin wax, and stained with luxol fast blue.

Western blotting analysis. The dorsal horns of the human postmortem lumbar and cervical spinal cord were dissected from sections on dry ice and homogenized in RIPA lysis buffer (1\% Nonidet P-40, 50 mm Tris$\mathrm{HCl}$, pH 7.4, $10 \%$ glycerol, $150 \mathrm{~mm} \mathrm{NaCl}, 1$ mm EDTA, pH 8.0) containing the protease inhibitor mixture (Sigma-Aldrich). After centrifugation $(12,000 \times g)$, the protein concentration in the supernatant was determined using the BCA Protein Assay Kit (Pierce). Equal amounts of protein $(50 \mu \mathrm{g})$ were loaded and separated by SDS-PAGE, followed by transferring to nitrocellulose membranes. The membranes were then blocked and incubated with anti-c-Fos (1:1000; Abcam; 16902), anti-
phospho-ERK1/2 (1:1000; Cell Signaling; 4370), anti-phospho-JNK (1: 1000; Cell Signaling; 9251), anti-phospho-p38 mitogen-activated protein kinase (MAPK) (1:1000; Cell Signaling; 9211), anti-phospho-cJun (1:1000; Cell Signaling; 9261), anti-phospho-CREB (1:1000; Cell Signaling; 9191), anti-TNF $\alpha$ (1:1000; Abcam; ab1793), anti-IL1 $\beta$ (1:1000; Santa Cruz; sc-7884), anti-CD11b (1:5000; Abcam; 52478), anti-Iba1 (1:2000; Wako; 016-20001), anti-glial fibrillary acidic protein (GFAP) (1:5000; Millipore; 04-1062), or anti-S100 $\beta$ (1:2000; Millipore; 04-1054) primary antibodies. Protein bands were visualized using the Enhanced Chemiluminescence kit (Pierce). Membranes were reblotted after stripping for multiple times without signal attenuation. $\beta$-Actin (1:1000; Santa Cruz Biotechnology; sc-1616-R) was blotted as loading controls.

Immunohistochemistry. Paraffin sections $(5 \mu \mathrm{m})$ of human lumbar spinal cords were deparaffinized in xylene and rehydrated in a series of ethanol $(100,95,80$, and $70 \%)$ and PBS, followed by incubation in 3\% $\mathrm{H}_{2} \mathrm{O}_{2}$ for 5-10 min to quench endogenous peroxidase or endogenous biotin activity. After antigen retrieval in sodium citrate buffer and blocking in 5\% BSA, the sections were incubated with anti-GFAP (1:500) or normal rabbit serum (negative control) for $1 \mathrm{~h}$ at room temperature. After washing for five times in TBST buffer (20 mM Tris-HCl, $150 \mathrm{~mm}$ $\mathrm{NaCl}, 0.1 \%$ Tween $20, \mathrm{pH} 8.0$ ), the sections were incubated with biotinlabeled secondary antibody and horseradish peroxidase-conjugated streptavidin. The sections were stained with DAB chromogen (Vector 
Table 2. Human patient treatment information

\begin{tabular}{|c|c|c|c|c|c|c|}
\hline Subject & NNTC ID ${ }^{a}$ & HIV infected & AIDS & AIDS-associated disease & HIV treatment & Other treatment \\
\hline 1 & 7101958083 & No & No & $\mathrm{N} / \mathrm{A}$ & $\mathrm{N} / \mathrm{A}$ & None \\
\hline 2 & 7102268283 & No & No & $\mathrm{N} / \mathrm{A}$ & $\mathrm{N} / \mathrm{A}$ & None \\
\hline 3 & 7101198377 & No & No & $\mathrm{N} / \mathrm{A}$ & N/A & $\begin{array}{l}\text { Adriamycin, bleomycin, vinblastine, } \\
\text { dacrobazine, levofloxacin, } \\
\text { cefepime, indomethazine }\end{array}$ \\
\hline 4 & 7101738266 & No & No & $\mathrm{N} / \mathrm{A}$ & $\mathrm{N} / \mathrm{A}$ & Antibiotics \\
\hline 5 & 7101727068 & No & No & $\mathrm{N} / \mathrm{A}$ & $\mathrm{N} / \mathrm{A}$ & Taxotere, prednisone, 0xyContin \\
\hline 6 & 7200188777 & Yes & Yes & Pneumocystic pneumonia & Unknown & Bactrim, Diflucan \\
\hline 7 & 7200586865 & Yes & Yes & $\begin{array}{l}\text { Polymicrobial pneumonitis, CNS } \\
\text { toxoplasmosis }\end{array}$ & Unknown & $\begin{array}{l}\text { Clindamycin, Daraprim, leucovorin, } \\
\text { vancomycin, Fortaz }\end{array}$ \\
\hline 8 & 7100567477 & Yes & Yes & Severe cachexia, bronchopneumonia & $\begin{array}{l}\text { 3TC, ABC, d4T, EFV, NFV, NVP, ZDV, } \\
\quad \text { AZT }\end{array}$ & $\begin{array}{l}\text { Dapsone, MS Contin, Dilaudid, } \\
\text { Valium, clonidine, Lasix, spi- } \\
\text { ronolactone, fentanyl patch }\end{array}$ \\
\hline 9 & 7200267172 & Yes & Yes & Severe cachexia, bronchopneumonia & $\begin{array}{l}\text { 3TC, ABC, d4T, SQV2 or FTV, NFV, } \\
\text { NVP, RTV }\end{array}$ & Unknown \\
\hline 10 & 7100696768 & Yes & Yes & Cytomegalovirus in bladder & $\begin{array}{l}\text { 3TC, ABC, d4T ddC, ddl, EFV, FTC, IDV, } \\
\quad \text { NFV, ZDV, AZT }\end{array}$ & $\begin{array}{l}\text { Pepcid, folic acid, Diflucan, Neu- } \\
\text { rontin, Senekot, and Megace }\end{array}$ \\
\hline 11 & 7100616568 & Yes & Yes & $\begin{array}{l}\text { Pneumocystis carinii pneumonia, } \\
\text { cachexia, HIVE }\end{array}$ & $\begin{array}{l}\text { 3TC, ABC, d4T, ddC, ddl, EFV, IDV, } \\
\text { NFV, NVP, ZDV, AZT }\end{array}$ & $\begin{array}{l}\text { MS Contin, Dilantin, dapsone, } \\
\text { quinapril, vecuronium, succinyl, } \\
\text { and Ativan }\end{array}$ \\
\hline 12 & 7100147470 & Yes & Yes & Unknown & EFV; RTV SQV & Unknown \\
\hline 13 & 7100766971 & Yes & Yes & Unknown & $\begin{array}{l}\text { 3TC, ABC, APV, d4T, EFV, IDV, NFV, } \\
\text { NVP, RTV, SQV, TFV, PMPA, ZDV, } \\
\text { AZT }\end{array}$ & $\begin{array}{l}\text { Lasix, spironolactone, Bactrim, } \\
\text { clonidine, zaithromycin }\end{array}$ \\
\hline 14 & 7100626868 & Yes & Yes & $\begin{array}{l}\text { Pneumocystis carinii pneumonia, } \\
\text { cachexia }\end{array}$ & Unknown & $\begin{array}{l}\text { Antihypertensives, anticonvulsants, } \\
\text { antidepressants, antihistamines, } \\
\text { opiates, antifungals }\end{array}$ \\
\hline 15 & 7100118382 & Yes & Yes & $\begin{array}{l}\text { Bronchopneumonia, epidermodysplasia } \\
\text { verruciformis }\end{array}$ & Unknown & Unknown \\
\hline
\end{tabular}

${ }^{a}$ NNTC ID, National NeuroAIDS Tissue Consortium identifier for public access.

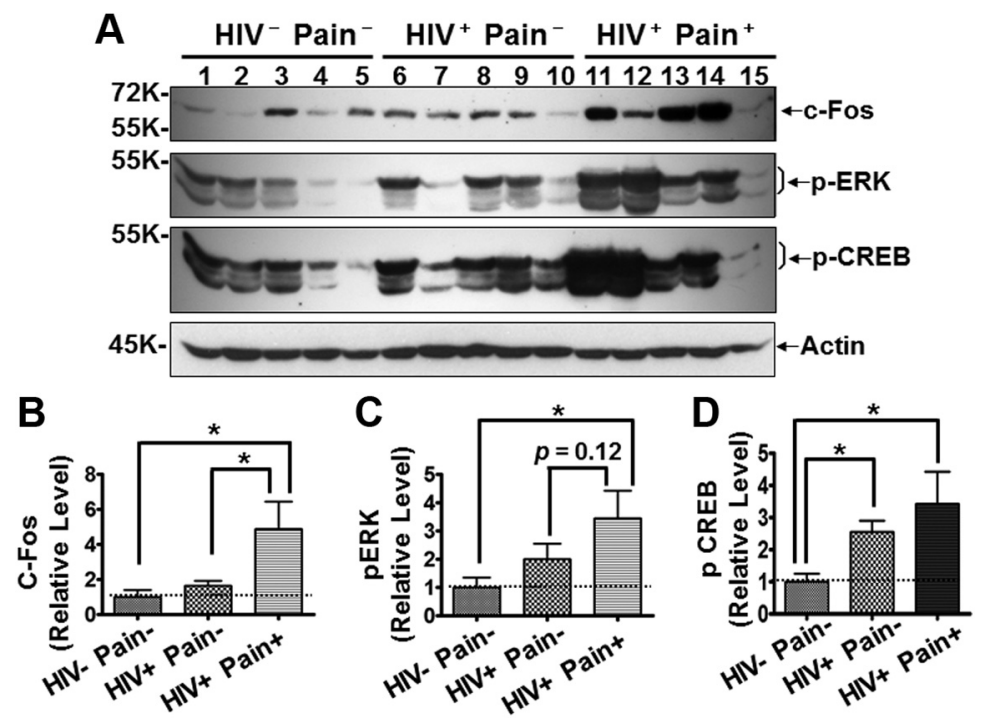

Figure 2. Levels of neuronal activity-regulated proteins in the SDH. A, Increased c-Fos, p-ERK, and p-CREB revealed by immunoblotting. Shown is the same membrane blotted with different antibodies after stripping; same for blots in other figures, unless indicated otherwise. $\boldsymbol{B}-\boldsymbol{D}$, Quantitative summary of $\mathrm{C}-\mathrm{FOS}(\boldsymbol{B}), \mathrm{p}$-ERK $(\boldsymbol{C})$, and $\mathrm{p}$-CREB $(\boldsymbol{D})$. Error bars indicate SEM. ${ }^{*} p<0.05$.

Laboratories). When DAB staining was finished, the sections were counterstained with hematoxylin to label nuclei.

Astrocyte counting. To facilitate astrocyte counting, the sections were counterstained with hematoxylin to label cell nuclei. Only GFAP-positive cells with clearly identifiable nuclei were counted. During cell counting, the images were zoomed in to help identify cells. Three different patients from each group (nonHIV and pain-negative or pain-positive HIV patients) and three sections from each patient were included in this analysis.
Data analysis and statistics. The protein bands on immunoblots were quantified using NIH ImageJ. $\beta$-Actin bands were used as loading controls. Values were recorded as mean \pm SEM from at least three independent experiments. One-way ANOVA was performed, using Prism 5 (GraphPad) software $(p<0.05$ was considered significant; ${ }^{*} p<0.05 ;{ }^{* *} p<$ 0.01).

\section{Results}

To survey the glial reaction profile in the $\mathrm{SDH}$, we used postmortem lumbar spinal cords from 15 sex- and age-matched human patients (Table 1). Five of them (numbers 1-5) were control patients who died from non-HIV/AIDS diseases and did not have chronic pain ( $\mathrm{HIV}^{-}$Pain $^{-}$). Another five (numbers 6-10) died with HIV-1 infection and AIDS, but they did not develop chronic pain ( $\mathrm{HIV}^{+} \mathrm{Pain}^{-}$). The rest (numbers 11-15) also died with HIV-1 infection and AIDS, but did develop the comorbidity of chronic pain $\left(\mathrm{HIV}^{+}\right.$Pain $\left.^{+}\right)$. The chronic pain was diagnosed either by the Cleeland Brief Pain Inventory or the NNTC Pain Diagnostic Worksheet. Clinically, the $\mathrm{HIV}^{+}$Pain $^{+}$patients were diagnosed with one or more pain symptoms in distal legs (Table 1). We were not able to obtain accurate information regarding the medication of pain management for these patients. When sural nerves were examined, the $\mathrm{HIV}^{+}$Pain $^{+}$patients manifested various degrees of neuropathy, judged from the de- 
crease of the density of myelinated axons (Fig. 1). All of the 10 HIV-infected patients developed AIDS. AIDS-related diseases, including pneumocystic pneumonia, polymicrobial pneumonitis, CNS toxoplasmosis, severe cachexia, bronchopneumonia, or epidermodysplasia verruciformis, were recorded with HIV-1/ AIDS patients (Table 2). Available information regarding the treatments the patients received was summarized in Table 2.

The reaction of microglia and astrocytes in the SDH is a cell hallmark for chronic pain in animal models and is believed to play a critical role in the development and/or maintenance of chronic pain. To test the hypothesis that glial reaction in the SDH is involved in the pathophysiology of pain associated with HDSPN, we performed Western blotting analysis of human lumbar spinal cord specimens. First, we determined whether the expression of neuronal activity-regulated proteins in the $\mathrm{SDH}$ was related to the diagnosis of pain. As shown in Figure $2 \mathrm{~A}$, c-Fos, a commonly used molecular marker of enhanced neuronal activity in the pain neural pathway in pain animal models (Coggeshall, 2005), was markedly increased in three of the five pain-positive HIV patients. Overall, the concentration of c-Fos protein was approximately fivefold higher in the pain-positive patients (Fig. $2 B$ ). The c-Fos concentration in pain-negative patients was not significantly different from that in the non-HIV patients (Fig. $2 A, B)$. Additional immunoblotting analyses on phosphorylated ERK (p-ERK) was done, which is often increased in the SDH of pain animal models (Ji et al., 1999; Gao and Ji, 2009). Similar to the c-Fos results, p-ERK was significantly increased in the SDH of the pain-positive HIV patients but not in the pain-negative HIV patients (Fig. 2A,C). ERKs are well known activity-regulated MAPKs and activate CREB in neurons (Davis et al., 2000; Song et al., 2005; Cao et al., 2009). Consistent with this notion, CREB phosphorylation (Ser133) was upregulated in the SDH of the pain-positive HIV patients (Fig. 2A,D). A less in magnitude but significant $\mathrm{p}$-CREB upregulation was also detected in the painnegative HIV patients (Fig. $2 A, D$ ), indicating that p-CREB upregulation is not necessarily associated with HIV chronic-pain state. It is important to point out that, in neuropathic pain models, p-ERK was also induced in microglia and astrocytes, especially in astrocytes (Zhuang et al., 2005). Generally, the results of upregulation of c-Fos and p-ERK found in the SDH of painpositive HIV patients were consistent to the findings in animal models. Notably, the increase of c-Fos and p-ERK was not uniformly observed in all pain-positive HIV patients. For instance, patient 15 did not show significant increase for either c-Fos or p-ERK (Fig. 2A). This was likely due to problems in protein quality from this particular patient, since the level of other proteins analyzed were often lower. Alternatively, this may indicate that the increase of c-Fos and p-ERK is not necessarily required for the pathogenesis of HIV-associated chronic pain.

Next, we determined the expression level of ionized calcium binding adaptor molecule 1 (Iba1), a protein upregulated in reactive microglia in animal models. Immunoblotting analysis showed that Ibal levels in the HIV-infected patients, including the pain-positive and pain-negative groups, were not significantly different from the levels in non-HIV patients (Fig. $3 A, B$ ). These data indicate that microglial cells did not increase in the SDH of HIV patients. To confirm this notion further, we determined the protein level of CD11b, a surface membrane protein that is upregulated in microglial cells when they are reactive. As shown in Figure 3, $A$ and $C, \mathrm{CD} 11 \mathrm{~b}$ did not increase in the SDH of either pain-negative or pain-positive HIV patients, compared with that in the non-HIV patients. Although Ibal and CD11b are also expressed in other cells in the monocyte lineage (e.g., mac-

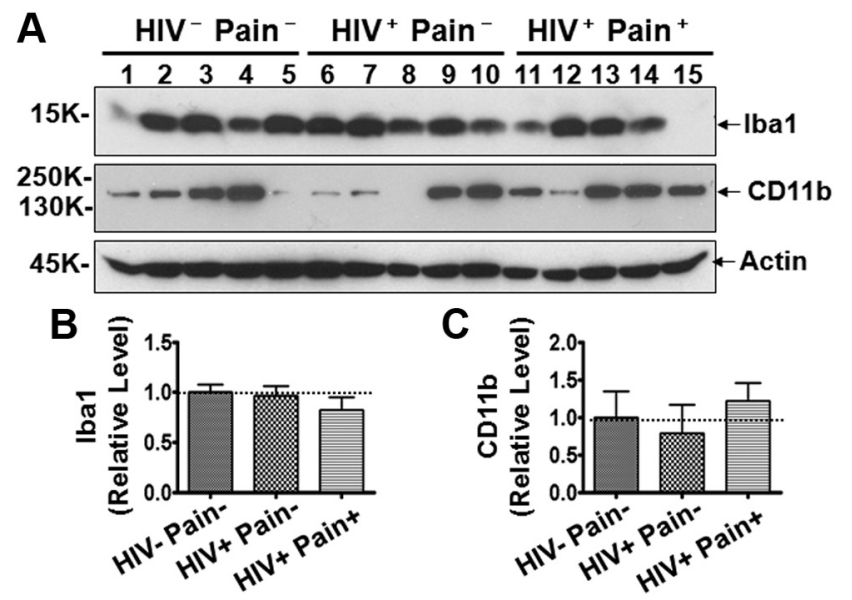

Figure 3. Expression of $\mathrm{Iba} 1$ and $C D 11 \mathrm{~b}$ in the SDH. $A$, Immunoblots of Iba1 and CD11b. $B, C$, Quantitative summary of Iba1 (B) and CD11b (C). Error bars indicate SEM.

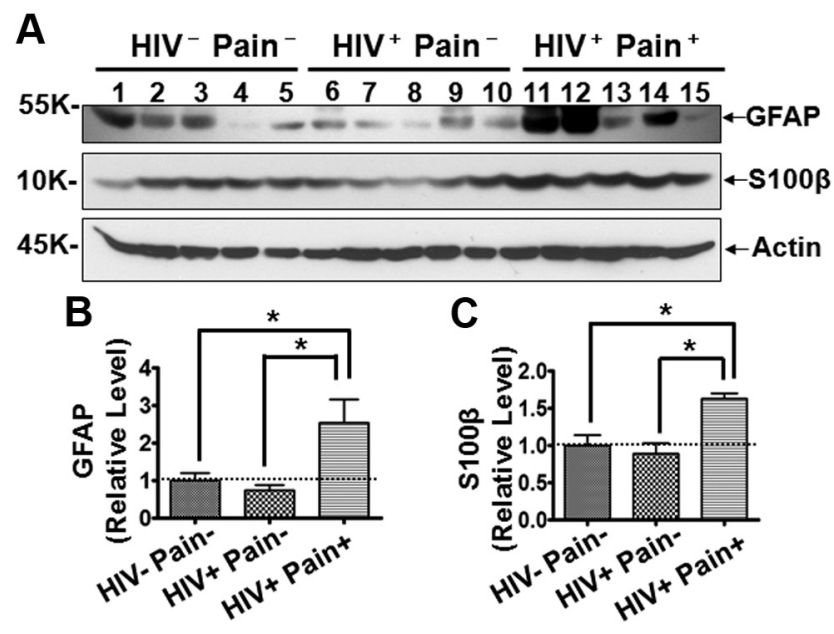

Figure 4. Levels of GFAP and S100 $\beta$ proteins in the SDH. $A$, Immunoblots of GFAP and $\mathrm{S} 100 \beta$. B, C, Quantitative summary of GFAP $(\boldsymbol{B})$ and $\mathrm{S} 100 \beta$ (C). Error bars indicate SEM. ${ }^{*} p<0.05$.

rophages) that may infiltrate the $\mathrm{SDH}$, these observations suggest that neither Iba1 nor CD11b-expressing microglia cells were increased in the lumbar SDH of the HIV-infected patients. Importantly, compared with the pain-negative HIV patients, both Iba1 and CD11b were not significantly increased in the pain-positive HIV patients (Fig. 3). This result indicates the possibility that the observed chronic pain state of the HIV patients may not be associated with microglial activation.

Because activated astrocytes are thought to play a critical role in chronic pain, especially during the maintenance phase (DeLeo et al., 2000; Ji et al., 2006; Zhuang et al., 2006; Gao et al., 2009, 2010a,b; Gao and Ji, 2010), we characterized astroglial reaction in the SDH of HIV patients. To this end, we determined the expression level of GFAP, an intermediate filament protein that is upregulated in reactive astroglia (Ridet et al., 1997). The result of immunoblotting analysis showed that GFAP was upregulated in the SDH of pain-positive HIV patients, whereas GFAP upregulation was not observed in the SDH of pain-negative HIV patients (Fig. 4A,B). These data are consistent with the idea that astrocyte reaction (indicated by GFAP increase) might be involved in the HIV-associated chronic pain. To test this notion more rigorously, we also characterized the expression of $\mathrm{S} 100 \beta$, an 


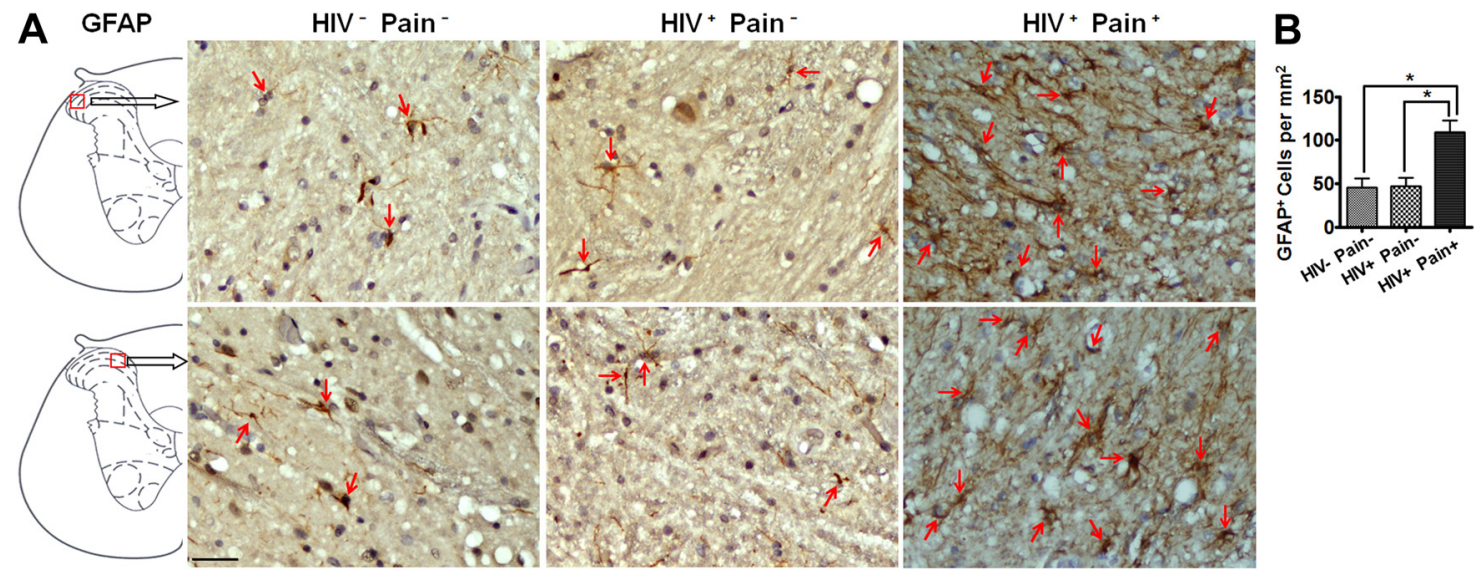

Figure 5. Increase of astrocytes in the SDH of pain-positive HIV patients revealed by immunohistochemistry.A, Representative images of GFAP staining from two different patients for each group. $B$, The density of GFAP-positive (brown) cells in the SDH. To facilitate cell counting, the sections were counterstained to label the nuclei (blue, arrows). Staining of the sections from the HIV ${ }^{-}$Pain ${ }^{-}$, $\mathrm{HIV}^{+}$Pain ${ }^{-}$, and HIV ${ }^{+}$Pain ${ }^{+}$patients was performed in parallel to keep the same experimental conditions among different groups. The dark staining of the HIV ${ }^{+}$Pain ${ }^{+}$sections was due to high GFAP expression. Scale bar, $50 \mu \mathrm{m}$. Error bars indicate SEM. ${ }^{*} p<0.05$.
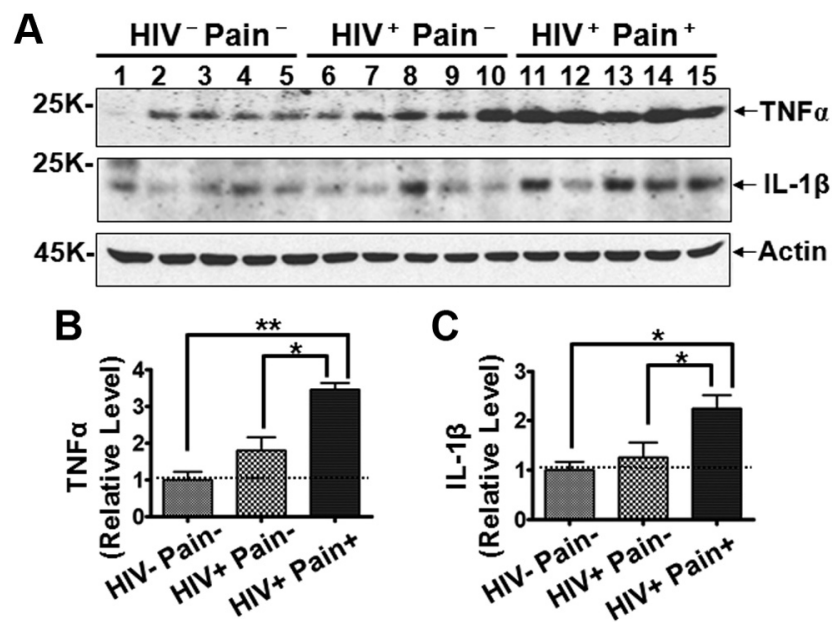

Figure 6. Expression levels of TNF $\alpha$ and IL-1 $\beta$ in the SDH. A, Immunoblots of TNF $\alpha$ and IL-1 $\beta . B, C$, Quantitative summary of TNF $\alpha(\boldsymbol{B})$ and IL-1 $\beta(\boldsymbol{C})$. Error bars indicateSEM. ${ }^{*} p<0.05$; ${ }^{* *} p<0.01$.
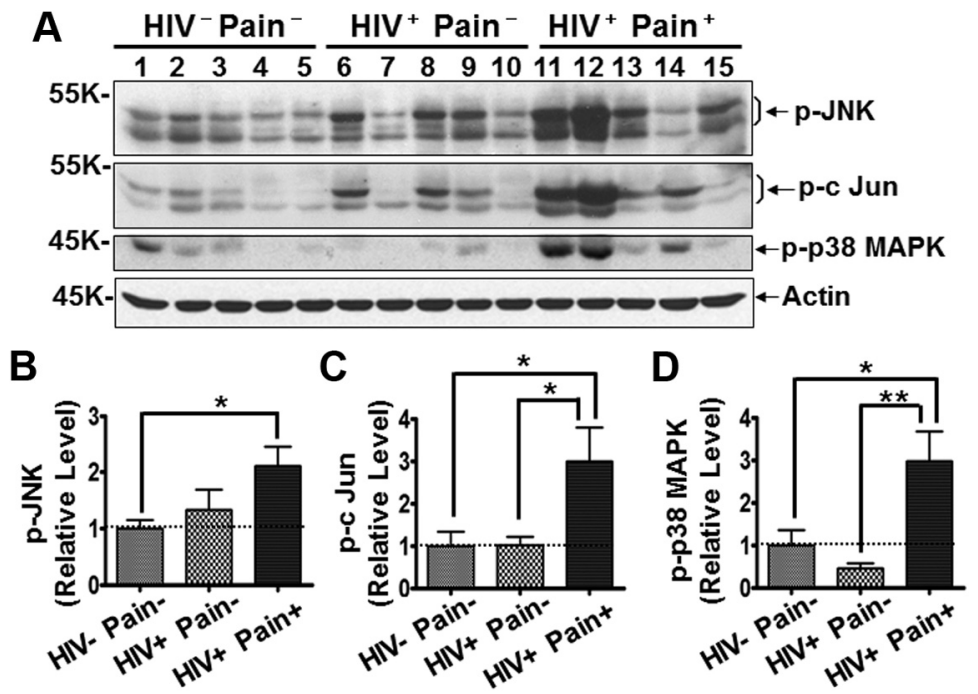

Figure 7. MAPK signaling in the SDH. $\boldsymbol{A}$, Immunoblots of phosphorylated JNK (p-JNK), phosphorylated c-Jun (p-c-Jun), and phosphorylated 38 ( $\mathrm{p}$-p38 MAPK). $\boldsymbol{B}-\boldsymbol{D}$, Quantitative summary of $\mathrm{p}-\mathrm{JNK}(\boldsymbol{B}), \mathrm{p}-\mathrm{c}-\mathrm{Jun}(\boldsymbol{C})$, and p-p38 MAPK (D). Error bars indicate SEM. ${ }^{*} p<0.05 ;{ }^{* *} p<0.01$. astroglial-derived calcium-binding protein that is upregulated during astrocyte reaction (Ridet et al., 1997). Similar to GFAP, $\mathrm{S} 100 \beta$ was also specifically upregulated in the SDH of the painpositive HIV patients but not the pain-negative HIV patients (Fig. $4 A-C$ ).

To further confirm the reaction of astrocytes in HIV patients, we performed immunohistochemistry analysis to compare the density and morphology of astrocytes in the lumbar SDH of different groups of patients. To this end, we performed GFAP immunohistochemistry of the SDH. The sections were counterstained with hematoxylin to reveal the nuclei. The results showed that GFAP-labeled cells were sparsely scattered in the SDH of non-HIV patients or pain-negative HIV patients (Fig. $5 A$ ). In contrast, GFAP-positive cells were more densely filled in the SDH of pain-positive HIV patients (Fig. 5A). When GFAP-positive cell bodies (revealed by hematoxylin counterstaining) were counted, we found that the density of GFAP-labeled astrocytes in the painpositive HIV patients was over twofold higher than that in the ether pain-negative HIV patients or non-HIV controls (Fig. 5B). The GFAP-positive cell density in the pain-negative HIV patients did not increase compared with non-HIV controls (Fig. 5B). In addition, GFAP-labeled astrocytes in the SDH of pain-positive HIV patients appeared to have a more complex branching organization, compared with their counterparts in non-HIV patients or pain-negative HIV patients (Fig. 5A), indicating that astrocytes in the SDH of pain-positive patients developed a hypertrophic morphology.

Activated glial cells are the main source of cellular mediators (e.g., cytokines, chemokines, and neurotrophins) that play important roles in chronic pain development in animal models (Oh et al., 2001; Milligan et al., 2003; Coull et al., 2005). Thus, we sought to determine the expression levels of TNF $\alpha$ and IL-1 $\beta$, two proinflammatory cytokines that are known to be produced in glial cells such as astrocytes (Fontana et al., 1982; Chung and Ben- 


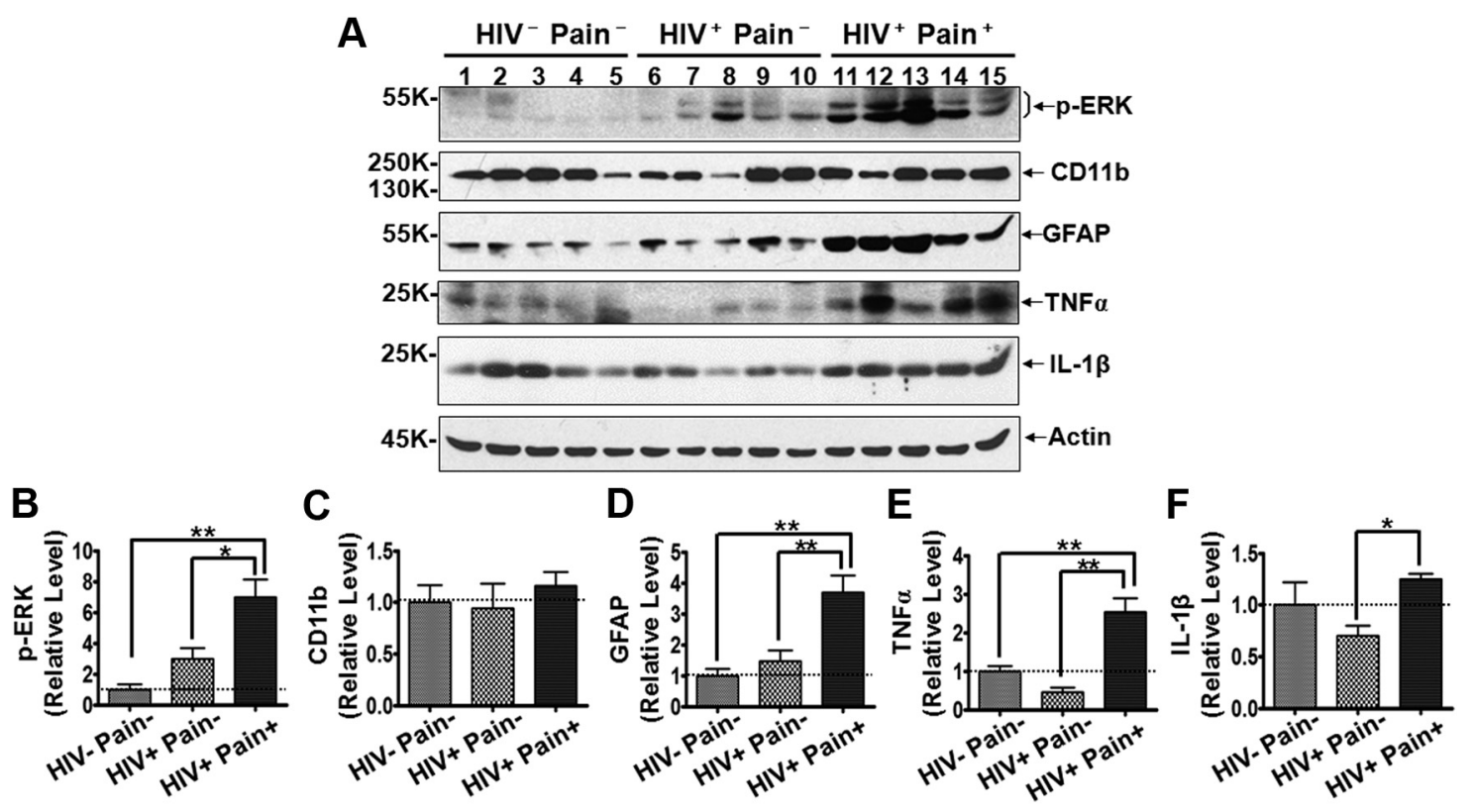

Figure 8. Characterization of molecular markers in the cervical SDH. A, Immunoblots of phosphorylated ERK (p-ERK), CD11b, GFAP, TNF $\alpha$, and IL-1 $\beta$. $\boldsymbol{B}-\boldsymbol{F}$, Quantitative summary of p-ERK (B), $\operatorname{CD11b}(\boldsymbol{C}), \operatorname{GFAP}(\boldsymbol{D}), \operatorname{TNF} \alpha(\boldsymbol{E})$, and IL- $1 \beta(\boldsymbol{F})$. Error bars indicate SEM. ${ }^{*} p<0.05 ;{ }^{* *} p<0.01$.

veniste, 1990). As shown in Figure 6, $A$ and $B$, the protein level of $\mathrm{TNF} \alpha$, a cytokine important for inflammatory and neuropathic pain (Cunha et al., 1992; Watkins et al., 1995a; Sommer et al., 2001; Schäfers et al., 2003), was higher in the SDH of painpositive HIV patients than that in the non-HIV patients $(\sim 3.5$-fold $)$, while this protein was not significantly upregulated in the pain-negative HIV patients. Similarly, IL- $1 \beta$ was also significantly upregulated in the SDH of pain-positive HIV patients but not in the pain-negative HIV patients (Fig. 6A$C)$. These findings support the idea that glial cells, likely astrocytes, were reactive in the SDH of the pain-positive HIV patients.

MAPKs, including ERK, JNK, and p38 MAPK, play essential roles in modulating chronic pain in animal models (Ji et al., 1999; Jin et al., 2003; Milligan et al., 2003; Gao and Ji, 2009; Gao et al., 2009). Our results described earlier showed that p-ERK was specifically activated in the SDH of pain-positive HIV patients (Fig. $2 A, C)$. It is important to point out that, in neuropathic pain models, p-ERK was induced not only in neurons but also in glia, especially astrocytes (Zhuang et al., 2005). More recent studies suggested relatively restrictive activation of JNKs in astrocytes in pain models (Gao et al., 2009, 2010a); we then compared the levels of p-JNKs in the SDH of different groups of the patients. We found that p-JNK (Thr183/Tyr185) significantly increased in the pain-positive but not the pain-negative HIV patients (Fig. $7 A, B)$. Similarly, phosphorylation of c-Jun (Ser63), a downstream target of the JNK signaling, was upregulated in the SDH of the pain-positive (but not the pain-negative) HIV patients (Fig. $7 A, C)$. These findings further support the idea of astrocyte reaction in the pain-positive HIV patients. The activation of $\mathrm{p} 38$ MAPK was thought to be restricted to microglia in animal pain models (Jin et al., 2003; Svensson et al., 2003). However, in contrast to the fact that Iba1 and CD11b were not upregulated in the $\mathrm{SDH}$ of the pain-positive patients, p38 MAPK (p-p38 MAPK, Thr180/Tyr182) was activated in the SDH of pain-positive (but not pain-negative) HIV patients (Fig. 7A,D). Because p38 MAPK was also reported to be activated in reactive astrocytes in the brain
(Che et al., 2001), it is possible that the reactive astrocytes in the $\mathrm{SDH}$ of the pain-positive HIV patients were the source of the observed p-p38 MAPK increase.

Overall, the data presented above appear to suggest the HIV pain-associated astrocyte reaction in the SDH at the lumbar level. There are different subtypes of astrocytes in different regions of the CNS (Hochstim et al., 2008; Oberheim et al., 2009), raising the possibility of differential HIV pain-related astrocyte reaction profiles in different regions in the spinal cord. To address this possibility, we performed Western blotting analysis of the cervical SDH. Similar results were obtained for various protein examined (Fig. 8). These data suggest that HIV pain-associated astrocyte reaction in the SDH likely occurs at different levels of the spinal cord.

\section{Discussion}

The data presented in this paper reveal for the first time that astrocytes were specifically reactive in the SDH of human HIV patients who developed chronic pain. In contrast, the astroglial reaction was not observed in the patients who did not suffer chronic pain. The observation of pain-associated reaction of astrocytes indicates that activated astrocytes may critically contribute to the maintenance of HIV-associated chronic pain. Consistent with this notion, studies on animal models have suggested that reaction of astrocytes plays a key role during the maintenance phase of pathological pain (Zhuang et al., 2006; Gao et al., 2009, 2010a,b). Notably, Zhang et al. (2012) reported that astrocytes but not microglia contribute to paclitaxel-induced neuropathic pain in rats. A recent study reveals multiple subtypes of cortical astrocytes in humans that manifest with unique morphologies and spatial distributions (Oberheim et al., 2009). Anatomically, some of them appear to be only in humans but not in rodents. It would be interesting to determine in future studies if the HIV pain-associated reaction of astrocytes is restricted to specific subtypes in the SDH.

Only some of HIV patients acquire the chronic pain state while others do not. The mechanism that determines who will 
transit to a chronic pain state is largely unknown. Although peripheral neuropathy is frequently $(\sim 30 \%)$ detected in painpositive HIV patients and often considered as a critical pathogenic event in the PNS, we currently understand little about the molecular and cellular processes in the CNS (especially SDH) that lead to the acquisition of HIV-associated chronic pain. The specific reaction of astrocytes in the SDH of pain-positive HIV patients and the critical role of astrocyte reaction in the establishment of chronic pain revealed by animal studies (Zhuang et al., 2006; Gao et al., 2009, 2010a,b) suggest a mechanistically intriguing possibility that the reaction of astrocytes in the $\mathrm{SDH}$ is an important step in the transition to HIV-associated chronic pain. It will be important to elucidate how the SDH astrocytes are reactive in the HIV patients. The observation that pain-negative HIV-infected patients did not show astrocyte hyper-reaction indicates that, in addition to HIV-related causes, host factors play a critical role in the astrocyte reaction. The pain-positive patients may have currently unknown host factors to facilitate the reaction of astrocytes.

Our results show that neither Iba1 nor CD11b are upregulated in the SDH of either pain-negative or pain-positive HIV patients (Fig. 3). Although these proteins are also expressed in macrophages, this finding indicates that they are not upregulated in the microglia in the SDH of the pain-positive HIV patients. In contrast, p-p38 MAPK, another protein whose phosphorylation is reported to be associated with microglial reaction (Jin et al., 2003; Svensson et al., 2003), is upregulated in the SDH of the painpositive but not pain-negative HIV patients (Fig. $7 A, D$ ). We cannot conclude at present whether microglia contribute to the observed upregulation of p-p38 MAPK in the SDH of the painpositive HIV patients, because neurons are a major source of this protein (Takeda and Ichijo, 2002). Despite this caveat, the Iba1 and CD11b data seem to indicate that microglia are not dramatically reactive. If this is true, it would suggest that microglial reaction in the $\mathrm{SDH}$ is not necessarily associated with the observed chronic pain state in the HIV patients. However, this interpretation does not exclude the possibility that microglial reaction participates in the maintenance of chronic pain in other clinical conditions. Indeed, Del Valle et al. (2009) reported microglial reaction in the SDH of one patient with longstanding regional pain syndrome, although confounding factors in that study were noted (McMahon and Malcangio, 2009). In addition, our finding does not exclude the potential involvement of reactive microglia during the initiation of HIV-associated chronic pain. Previous studies on animal models suggested that microglia in the SDH became reactive after of the induction of chronic pain but the microglial reaction was relatively transient (Milligan et al., 2001) (but see Clark et al., 2007a). Thus, it is possible that microglia were transiently activated during the early phase of HIV-associated pain development.

One view, based on animal model studies, is that during chronic pain development microglia release cytokines and/or chemokines to activate astrocytes. The potential dissociation of astrocyte and microglial reaction in the SDH of pain-positive HIV patients shown in this study suggests that microglial reaction is not required for the maintenance of astrocyte reaction. It is unclear whether activated microglia were involved in the initiation of astrocyte reaction in these HIV patients. More recent studies with pain models also revealed that microglial reaction and astrocyte reaction are not always coupled. For example, astrocyte reaction was observed in some chronic pain models without microglial reaction (Colburn and DeLeo, 1999; Honore et al., 2000; Hald et al., 2009) and microglial reaction was not associated with concurrent or subsequent astroglial reaction in other models (Svensson and Brodin, 2010).

In summary, results from this study provide evidence for the specific reaction of glial cells, particularly astrocytes, in the SDH of human HIV patients who developed chronic pain. This finding suggests that astroglial reaction is likely involved in the pathogenesis of HIV-associated chronic pain. It would be interesting to determine whether blockage of astroglial reaction can prevent or reverse the chronic pain experienced by HIV patients.

\section{References}

Cao H, Gao YJ, Ren WH, Li TT, Duan KZ, Cui YH, Cao XH, Zhao ZQ, Ji RR, Zhang YQ (2009) Activation of extracellular signal-regulated kinase in the anterior cingulate cortex contributes to the induction and expression of affective pain. J Neurosci 29:3307-3321.

Che Y, Yu YM, Han PL, Lee JK (2001) Delayed induction of p38 MAPKs in reactive astrocytes in the brain of mice after KA-induced seizure. Brain Res Mol Brain Res 94:157-165.

Chung IY, Benveniste EN (1990) Tumor necrosis factor- $\alpha$ production by astrocytes. Induction by lipopolysaccharide, IFN- $\gamma$, and IL- $1 \beta$. J Immunol 144:2999-3007.

Clark AK, Gentry C, Bradbury EJ, McMahon SB, Malcangio M (2007a) Role of spinal microglia in rat models of peripheral nerve injury and inflammation. Eur J Pain 11:223-230.

Clark AK, Yip PK, Grist J, Gentry C, Staniland AA, Marchand F, Dehvari M, Wotherspoon G, Winter J, Ullah J, Bevan S, Malcangio M (2007b) Inhibition of spinal microglial cathepsin $\mathrm{S}$ for the reversal of neuropathic pain. Proc Natl Acad Sci U S A 104:10655-10660.

Coggeshall RE (2005) Fos, nociception and the dorsal horn. Prog Neurobiol 77:299-352.

Colburn RW, DeLeo JA (1999) The effect of perineural colchicine on nerve injury-induced spinal glial activation and neuropathic pain behavior. Brain Res Bull 49:419-427.

Coull JA, Beggs S, Boudreau D, Boivin D, Tsuda M, Inoue K, Gravel C, Salter MW, De Koninck Y (2005) BDNF from microglia causes the shift in neuronal anion gradient underlying neuropathic pain. Nature 438:1017-1021

Cunha FQ, Poole S, Lorenzetti BB, Ferreira SH (1992) The pivotal role of tumour necrosis factor alpha in the development of inflammatory hyperalgesia. Br J Pharmacol 107:660-664.

Davis S, Vanhoutte P, Pages C, Caboche J, Laroche S (2000) The MAPK/ ERK cascade targets both Elk-1 and cAMP response element-binding protein to control long-term potentiation-dependent gene expression in the dentate gyrus in vivo. J Neurosci 20:4563-4572.

DeLeo JA, Rutkowski MD, Stalder AK, Campbell IL (2000) Transgenic expression of TNF by astrocytes increases mechanical allodynia in a mouse neuropathy model. Neuroreport 11:599-602.

Del Valle L, Schwartzman RJ, Alexander G (2009) Spinal cord histopathological alterations in a patient with longstanding complex regional pain syndrome. Brain Behav Immun 23:85-91.

Evers S, Wibbeke B, Reichelt D, Suhr B, Brilla R, Husstedt I (2000) The impact of HIV infection on primary headache. Unexpected findings from retrospective, cross-sectional, and prospective analyses. Pain 85:191-200.

Fontana A, Kristensen F, Dubs R, Gemsa D, Weber E (1982) Production of prostaglandin $\mathrm{E}$ and an interleukin-1 like factor by cultured astrocytes and C6 glioma cells. J Immunol 129:2413-2419.

Gao YJ, Ji RR (2009) c-Fos and pERK, which is a better marker for neuronal activation and central sensitization after noxious stimulation and tissue injury? Open Pain J 2:11-17.

Gao YJ, Ji RR (2010) Targeting astrocyte signaling for chronic pain. Neurotherapeutics 7:482-493.

Gao YJ, Zhang L, Samad OA, Suter MR, Yasuhiko K, Xu ZZ, Park JY, Lind AL, Ma Q, Ji RR (2009) JNK-induced MCP-1 production in spinal cord astrocytes contributes to central sensitization and neuropathic pain. J Neurosci 29:4096-4108.

Gao YJ, Xu ZZ, Liu YC, Wen YR, Decosterd I, Ji RR (2010a) The c-Jun $\mathrm{N}$-terminal kinase 1 (JNK1) in spinal astrocytes is required for the maintenance of bilateral mechanical allodynia under a persistent inflammatory pain condition. Pain 148:309-319.

Gao YJ, Zhang L, Ji RR (2010b) Spinal injection of TNF- $\alpha$-activated astro- 
cytes produces persistent pain symptom mechanical allodynia by releasing monocyte chemoattractant protein-1. Glia 58:1871-1880.

Garrison CJ, Dougherty PM, Kajander KC, Carlton SM (1991) Staining of glial fibrillary acidic protein (GFAP) in lumbar spinal cord increases following a sciatic nerve constriction injury. Brain Res 565:1-7.

Hald A, Nedergaard S, Hansen RR, Ding M, Heegaard AM (2009) Differential activation of spinal cord glial cells in murine models of neuropathic and cancer pain. Eur J Pain 13:138-145.

Hewitt DJ, McDonald M, Portenoy RK, Rosenfeld B, Passik S, Breitbart W (1997) Pain syndromes and etiologies in ambulatory AIDS patients. Pain 70:117-123.

Hochstim C, Deneen B, Lukaszewicz A, Zhou Q, Anderson DJ (2008) Identification of positionally distinct astrocyte subtypes whose identities are specified by a homeodomain code. Cell 133:510-522.

Honore P, Rogers SD, Schwei MJ, Salak-Johnson JL, Luger NM, Sabino MC, Clohisy DR, Mantyh PW (2000) Murine models of inflammatory, neuropathic and cancer pain each generates a unique set of neurochemical changes in the spinal cord and sensory neurons. Neuroscience 98: 585-598.

Ji RR, Baba H, Brenner GJ, Woolf CJ (1999) Nociceptive-specific activation of ERK in spinal neurons contributes to pain hypersensitivity. Nat Neurosci 2:1114-1119.

Ji RR, Kawasaki Y, Zhuang ZY, Wen YR, Decosterd I (2006) Possible role of spinal astrocytes in maintaining chronic pain sensitization: review of current evidence with focus on $\mathrm{bFGF/JNK}$ pathway. Neuron Glia Biol 2:259-269.

Jin SX, Zhuang ZY, Woolf CJ, Ji RR (2003) p38 mitogen-activated protein kinase is activated after a spinal nerve ligation in spinal cord microglia and dorsal root ganglion neurons and contributes to the generation of neuropathic pain. J Neurosci 23:4017-4022.

Kawasaki Y, Zhang L, Cheng JK, Ji RR (2008) Cytokine mechanisms of central sensitization: distinct and overlapping role of interleukin- $1 \beta$, interleukin-6, and tumor necrosis factor- $\alpha$ in regulating synaptic and neuronal activity in the superficial spinal cord. J Neurosci 28:5189-5194.

Ledeboer A, Sloane EM, Milligan ED, Frank MG, Mahony JH, Maier SF, Watkins LR (2005) Minocycline attenuates mechanical allodynia and proinflammatory cytokine expression in rat models of pain facilitation. Pain 115:71-83.

Ledeboer A, Liu T, Shumilla JA, Mahoney JH, Vijay S, Gross MI, Vargas JA, Sultzbaugh L, Claypool MD, Sanftner LM, Watkins LR, Johnson KW (2006) The glial modulatory drug AV411 attenuates mechanical allodynia in rat models of neuropathic pain. Neuron Glia Biol 2:279-291.

Ledeboer A, Hutchinson MR, Watkins LR, Johnson KW (2007) Ibudilast (AV-411). A new class therapeutic candidate for neuropathic pain and opioid withdrawal syndromes. Expert Opin Investig Drugs 16:935-950.

McMahon SB, Malcangio M (2009) Current challenges in glia-pain biology. Neuron 64:46-54.

Meller ST, Dykstra C, Grzybycki D, Murphy S, Gebhart GF (1994) The possible role of glia in nociceptive processing and hyperalgesia in the spinal cord of the rat. Neuropharmacology 33:1471-1478.

Milligan ED, Watkins LR (2009) Pathological and protective roles of glia in chronic pain. Nat Rev Neurosci 10:23-36.

Milligan ED, O'Connor KA, Nguyen KT, Armstrong CB, Twining C, Gaykema RP, Holguin A, Martin D, Maier SF, Watkins LR (2001) Intrathecal HIV-1 envelope glycoprotein gp120 induces enhanced pain states mediated by spinal cord proinflammatory cytokines. J Neurosci 21: $2808-2819$.

Milligan ED, Twining C, Chacur M, Biedenkapp J, O'Connor K, Poole S, Tracey K, Martin D, Maier SF, Watkins LR (2003) Spinal glia and proinflammatory cytokines mediate mirror-image neuropathic pain in rats. J Neurosci 23:1026-1040.

Mirsattari SM, Power C, Nath A (1999) Primary headaches in HIV-infected patients. Headache 39:3-10.

Morgello S, Gelman BB, Kozlowski PB, Vinters HV, Masliah E, Cornford M, Cavert W, Marra C, Grant I, Singer EJ (2001) The National NeuroAIDS Tissue Consortium: a new paradigm in brain banking with an emphasis on infectious disease. Neuropathol Appl Neurobiol 27:326-335.

Morgello S, Estanislao L, Simpson D, Geraci A, DiRocco A, Gerits P, Ryan E, Yakoushina T, Khan S, Mahboob R, Naseer M, Dorfman D, Sharp V; Manhattan HIV Brain Bank (2004) HIV-associated distal sensory polyneuropathy in the era of highly active antiretroviral therapy: The Manhattan HIV Brain Bank. Arch Neurol 61:546-551.
Oberheim NA, Takano T, Han X, He W, Lin JH, Wang F, Xu Q, Wyatt JD, Pilcher W, Ojemann JG, Ransom BR, Goldman SA, Nedergaard M (2009) Uniquely hominid features of adult human astrocytes. J Neurosci 29:3276-3287.

Oh SB, Tran PB, Gillard SE, Hurley RW, Hammond DL, Miller RJ (2001) Chemokines and glycoprotein 120 produce pain hypersensitivity by directly exciting primary nociceptive neurons. J Neurosci 21:5027-5035.

Ren K, Dubner R (2010) Interactions between the immune and nervous systems in pain. Nat Med 16:1267-1276.

Ridet JL, Malhotra SK, Privat A, Gage FH (1997) Reactive astrocytes: cellular and molecular cues to biological function. Trends Neurosci 20:570-577.

Rolan P, Gibbons JA, He L, Chang E, Jones D, Gross MI, Davidson JB, Sanftner LM, Johnson KW (2008) Ibudilast in healthy volunteers: safety, tolerability and pharmacokinetics with single and multiple doses. $\mathrm{Br} \mathrm{J}$ Clin Pharmacol 66:792-801.

Rolan P, Hutchinson M, Johnson K (2009) Ibudilast: a review of its pharmacology, efficacy and safety in respiratory and neurological disease. Expert Opin Pharmacother 10:2897-2904.

Romero-Sandoval EA, Horvath RJ, DeLeo JA (2008) Neuroimmune interactions and pain: focus on glial-modulating targets. Curr Opin Investig Drugs 9:726-734

Schäfers M, Lee DH, Brors D, Yaksh TL, Sorkin LS (2003) Increased sensitivity of injured and adjacent uninjured rat primary sensory neurons to exogenous tumor necrosis factor- $\alpha$ after spinal nerve ligation. J Neurosci 23:3028-3038.

Singer EJ, Zorilla C, Fahy-Chandon B, Chi S, Syndulko K, Tourtellotte WW (1993) Painful symptoms reported by ambulatory HIV-infected men in a longitudinal study. Pain 54:15-19.

Sommer C, Lindenlaub T, Teuteberg P, Schäfers M, Hartung T, Toyka KV (2001) Anti-TNF-neutralizing antibodies reduce pain-related behavior in two different mouse models of painful mononeuropathy. Brain Res 913:86-89.

Song XS, Cao JL, Xu YB, He JH, Zhang LC, Zeng YM (2005) Activation of ERK/CREB pathway in spinal cord contributes to chronic constrictive injury-induced neuropathic pain in rats. Acta Pharmacol Sin 26:789-798.

Svensson CI, Brodin E (2010) Spinal astrocytes in pain processing: nonneuronal cells as therapeutic targets. Mol Interv 10:25-38.

Svensson CI, Marsala M, Westerlund A, Calcutt NA, Campana WM, Freshwater JD, Catalano R, Feng Y, Protter AA, Scott B, Yaksh TL (2003) Activation of p38 mitogen-activated protein kinase in spinal microglia is a critical link in inflammation-induced spinal pain processing. J Neurochem 86:1534-1544.

Takeda K, Ichijo H (2002) Neuronal p38 MAPK signalling: an emerging regulator of cell fate and function in the nervous system. Genes Cells 7:1099-1111.

Wallace VC, Blackbeard J, Pheby T, Segerdahl AR, Davies M, Hasnie F, Hall S, McMahon SB, Rice AS (2007) Pharmacological, behavioural and mechanistic analysis of HIV-1 gp120 induced painful neuropathy. Pain 133:47-63.

Watkins LR, Goehler LE, Relton J, Brewer MT, Maier SF (1995a) Mechanisms of tumor necrosis factor-alpha (TNF-alpha) hyperalgesia. Brain Res 692:244-250.

Watkins LR, Deak T, Silbert L, Martinez J, Goehler L, Relton J, Martin D, Maier SF (1995b) Evidence for involvement of spinal cord glia in diverse models of hyperalgesia. Soc Neurosci Abstr 21:879.

Watkins LR, Martin D, Ulrich P, Tracey KJ, Maier SF (1997) Evidence for the involvement of spinal cord glia in subcutaneous formalin induced hyperalgesia in the rat. Pain 71:225-235.

Zhang H, Yoon SY, Zhang H, Dougherty PM (2012) Evidence that spinal astrocytes but not microglia contribute to the pathogenesis of Paclitaxelinduced painful neuropathy. J Pain 13:293-303.

Zhuang ZY, Gerner P, Woolf CJ, Ji RR (2005) ERK is sequentially activated in neurons, microglia, and astrocytes by spinal nerve ligation and contributes to mechanical allodynia in this neuropathic pain model. Pain 114:149-159.

Zhuang ZY, Wen YR, Zhang DR, Borsello T, Bonny C, Strichartz GR, Decosterd I, Ji RR (2006) A peptide c-Jun N-terminal kinase (JNK) inhibitor blocks mechanical allodynia after spinal nerve ligation: respective roles of JNK activation in primary sensory neurons and spinal astrocytes for neuropathic pain development and maintenance. J Neurosci 26:35513560 . 